\title{
La configuración de la enseñanza y aprendizaje de la historia como campo de conocimiento
}

\section{The shaping of the teaching and learning of history as a field of knowledge}

DOI: https://doi.org/10.32870/dse.v0i22.737

\author{
Citalic López Aceves* \\ María Guadalupe García Alcaraz**
}

\begin{abstract}
Resumen
En este documento presentamos un análisis sobre la configuración del campo de investigación en enseñanza de la historia, para lo cual ubicamos tres grandes tendencias. En la primera, la psicología permeó las explicaciones y las propuestas; en la segunda, los investigadores plantearon llevar el conocimiento histórico a las aulas imitando los procedimientos de los historiadores, mediación que corrió a cargo de la didáctica, además, se interesaron por comprender por qué las reformas y propuestas no se hacían efectivas en la escuela. Finalmente, en la actualidad observamos una bifurcación entre la educación histórica cuyo núcleo de interés está en el desarrollo del pensamiento histórico considerando los contextos locales, y la investigación en enseñanza de la historia que postula inscribir la producción de este conocimiento en el marco de los debates de las ciencias sociales.
\end{abstract}

Palabras clave: Enseñanza de la historia - psicología - pedagogía - historia - didáctica.

\begin{abstract}
In this document we present an analysis of the shaping of the field of research in the teaching of history, where we can locate three major trends. In the first one of them, psychology permeated the explanations and proposals. In the second one, researchers proposed bringing historical knowledge into the classroom by imitating the procedures of historians, mediated by the didactics, and were also interested in understanding why the reforms and proposals were not made effective in the school. Finally, at present we observe a bifurcation between historical education, whose nucleus of interest is in the development of historical thought considering local contexts, and research in history teaching that proposes inscribing the production of this knowledge within the framework of the social science debates.
\end{abstract}

Keywords: Teaching of History - Psychology - Pedagogy - History - Didactic.

\footnotetext{
* Maestra en Historia del mundo hispánico y maestrante en Desarrollo Social. Profesora de asignatura del Departamento de Humanidades de la Universidad Panamericana-Guadalajara. México. cilopez@up.edu.mx

** Doctora en Ciencias de la Educación. Profesora-investigadora titular "C". Departamento de Estudios en Educación, Universidad de Guadalajara. México. mggarcia.alcaraz@gmail.com
} 


\section{Introducción}

Nuestro interés por el tema que tratamos en este documento surgió de un señalamiento hecho por Paulina Latapí (2014) acerca de que la investigación en el campo de la enseñanza de la historia se ha desarrollado con poca integración, por lo que pensamos que un esfuerzo de revisión y síntesis permitirá avanzar en ese sentido.

Con base en lo señalado por Josefina Granja (2000), hacemos uso del concepto de configuración adoptando una postura analítica que pretende comprender las continuidades, desplazamientos y reorganizaciones experimentadas en el tiempo en torno a un conjunto de elaboraciones teóricas y metodológicas construidas por un grupo de académicos, mediante las cuales nombran, explican y comprenden cierto segmento de la realidad. Dar cuenta de este proceso implica elaborar un campo relacional entre productores, productos y receptores, en una dinámica en la que circulan y se relaboran ideas y propuestas en situaciones de tensión, desplazamiento o condensación, todo lo cual configura, justamente, un campo del conocimiento. Esta perspectiva nos permite sostener que, como parte de la complejidad y la particularidad de hacer un estado del conocimiento sobre la enseñanza y el aprendizaje de la historia, existen vasos comunicantes entre el desarrollo de las perspectivas y la generación de políticas de enseñanza y propuestas didácticas. Lo cual sucede así porque en este campo de conocimiento se trabaja con saberes que sufren adecuaciones para ser escolarizados.

Para delinear los contornos de este trabajo definimos dos ejes de análisis: $a$ ) los enfoques para la enseñanza de la historia desde la psicología educativa, la didáctica y la historia; y b) las propuestas que han emanado de esos enfoques y algunos de sus efectos en los modelos de formación docente, en los diseños curriculares y en la generación materiales educativos. Estos hilos conductores se entretejen en tres etapas, mismas que sirvieron para estructurar la exposición: la primera trata sobre la gestación del campo en enseñanza de la historia en el que predominó la psicología genética; en la segunda etapa ubicamos cómo fue que desde la didáctica, a partir de la década de los ochenta se construyeron puentes entre la teoría psicológica y la enseñanza en el aula; en la tercera exponemos los debates y enfoques recientes acerca de lo que se denomina educación histórica e investigación en enseñanza de la historia.

Metodológicamente, revisamos publicaciones en libros y revistas especializadas en torno a las perspectivas elaboradas desde mediados del siglo XX hasta la actualidad, desde tres núcleos de producción: psicología educativa, didáctica e historia, para desde ahí comprender las implicaciones que dichas investigaciones han tenido en algunas propuestas de enseñanza. En cuanto a la dimensión espacio-temporal de este trabajo, nuestro anclaje consistió en pensar cómo, cuándo y dónde se generaron conocimientos en torno a la enseñanza-aprendizaje de la historia, para luego discernir el posible impacto que tuvieron en México.

Diálo@os año 12 | número 22 | enero-junio 2021 | ISSN 2007-2171 


\section{La gestación (1960-1980)}

Para Sebastián Plá (2012), el campo de investigación en la enseñanza de la historia inició a partir de los postulados de Piaget acerca del desarrollo de las nociones temporales en el niño. El desarrollo consistía en una sucesión de estadios ${ }^{1}$ asociados a un aumento paulatino de la complejidad explicativa en los sujetos (Fairsten, Carretero, 2001: 181). Hervás y Miralles (2006) señalan que con base en esta teoría genética fue posible reflexionar sobre la temporalidad, en tanto noción fundamental para la enseñanza de la historia. Se argumentó que dicha noción formaba parte de un pensamiento complejo asociado a procesos de restructuración sucesiva, vinculados a las etapas madurativas del individuo. Con base en lo anterior, se estableció que la maduración cognitiva, en relación con la temporalidad, se alcanzaría casi de manera "natural"y el aprendizaje del contenido sería la manera directa de formar ese tipo de pensamiento.

Con el tiempo se reconoció que las investigaciones realizadas con esta perspectiva tuvieron algunos sesgos, en tanto se partió de una lectura casi literal de las etapas del desarrollo cognitivo, y los estudios revelaban que muy pocos niños alcanzaban el dominio de las nociones temporales "adecuadas" a su edad. Esto se debió, según Santiesteban (2017), a que las ideas de Piaget se trasladaron a las aulas de manera mecánica, sin tomar en cuenta otros factores cualitativos en el aprendizaje, por ejemplo, lo subjetivo de las nociones temporales en relación con cómo viven las personas, lo cual implica considerar que los conceptos elaborados acerca del tiempo están encuadrados por una diversidad de contextos y experiencias.

Los cuestionamientos antes señalados se relacionan con los debates iniciados en las conferencias de Woods Hole, llevadas a cabo en 1959 en el Instituto Tecnológico de Massachusetts. Allí se discutió la relación entre desarrollo y estadios evolutivos, preocupación abanderada por los delegados de Europa; el segundo tema, que inquietaba a los delegados de Estado Unidos, era ¿cómo acelerar las etapas de desarrollo? ${ }^{2}$ En ambos casos hubo cuestionamientos hacia las teorías piagetanas o a la manera como se hizo uso de ellas de forma casi literal, por lo que se estableció la necesidad de pensar no solo en el desarrollo evolutivo natural del niño sino en la influencia del profesor y del currículo. Estas puntualizaciones corrieron a la par de los despla-

1 Linares (2007) sintetiza las cuatro etapas de desarrollo cognitivo propuestas por Jean Piaget, mismas que sirven para explicar cómo los niños construyen un modelo mental del mundo. La primera etapa es la sensoriomotora, que va del nacimiento a los 2 años de vida, se caracteriza por el egocentrismo extremo, la idea de la permanencia de los objetos y el pensamiento orientado a medios y fines. La siguiente etapa es la preoperacional, de los 2 a los 7 años, el niño es capaz de usar símbolos y palabras para pensar y ponerse en el lugar del otro, pero mantiene la rigidez del pensamiento y el egocentrismo. La tercera etapa, de las operaciones concretas, va de los 7 a los 12 años, su rasgo central es el desarrollo del pensamiento racional y organizado. La última etapa es la de operaciones formales, desde los 12 años hasta la vida adulta, aparece y se consolida el razonamiento abstracto, el uso de la lógica científica, proposicional y proporcional. Para el tema que nos interesa, las nociones de tiempo y espacio se desarrollan de manera paulatina durante las cuatro etapas.

2 Mientras que los seguidores de Piaget trataban de respetar casi al pie de la letra las cuatro etapas de desarrollo cognitivo, la escuela estadunidense, encabezada por Jerome Bruner, abogaba por la posibilidad de su aceleración mediante agentes externos como el profesor y el currículo (Duckword, 1981). En ese marco, Bruner publicó su libro A Study of Thinking y para 1960 conformó el Centro de Estudios Cognitivos en Harvard, junto con Miller. Entre los temas que analizaron se encontraba la memoria, la formación de conceptos, el pensamiento y la teoría evolutiva (Díaz, 1999).

Diálopos sobre Educación 
zamientos hacia el cognitivismo, corriente de pensamiento que se desarrolló en el contexto de la guerra fría. Los gobiernos apoyaron estas investigaciones con una lógica política, buscando acelerar la formación de una fuerza intelectual que incidiera en el desarrollo económico (Díaz, 1999).

Ahora bien, ¿cuál es la influencia de ambas posturas en la enseñanza de la historia? La postura estadunidense, representada por Jerome Bruner, dio pie a la revolución cognitiva. Él planteó que el "alumno no debe hablar de física, historia o matemáticas [...] sino hacer física, historia, matemáticas [ya que] el conocimiento verdaderamente adquirido es aquel que se redescubre" (Bruner, 1963: 247, citado en Guilar, 2009: 23). Desde esta perspectiva, el aprendizaje se definió como un proceso activo, de asociación, construcción y representación.

Los aprendices deberían experimentar y emular el trabajo de los científicos mediante un currículo en espiral, ${ }^{3}$ que poco a poco se profundizaría en el conocimiento en función de la ampliación de la comprensión y capacidad cognitiva. Por ello, Bruner (1963) propuso llevar la metodología de las ciencias a la enseñanza en las aulas mediante el método por descubrimiento, que consistía en trabajar el contenido de las asignaturas imitando los procedimientos de los científicos.

Mientras tanto, en Europa hubo interés por entender el paso de un estadio a otro, profundizando en la compresión del proceso implicado. Esta inquietud llevó a intelectuales como Dennis Shemilt, en Inglaterra en 1972, a buscar una relación entre las posturas evolutivas y el desarrollo cognitivo (Duckworth, 1981). La intención era indagar "cómo el ser humano construye el conocimiento en el contexto de la interacción individuo-medio: [...] cómo crecen los conocimientos, cómo se transforman mediante ajustes progresivos y cómo estos siguen una progresión de un nivel inferior a uno superior, es decir de un pensamiento sensomotor al pensamiento formal" (Díaz, 1999: 217).

Desde esta postura se realizaron investigaciones que indagaban si en la adolescencia los estudiantes eran capaces de entender ciertas nociones de la disciplina al emular el trabajo de los historiadores. Así, se diseñaron propuestas curriculares que incluían la epistemología histórica y su metodología con la finalidad de que los alumnos comprendieran tanto la lógica como los métodos y perspectivas de la disciplina (Lamoneda, 1988). Para el diseño de estas propuestas se retomaron principios de la Escuela Francesa de los Annales: la historia problema, el empleo de fuentes primarias diversas, cierto abandono de la historia política y el arribo de temas sociales y económicos. ${ }^{4}$

3 Con esta premisa se llegó a afirmar que se puede enseñar cualquier tema a un niño, siempre que se respeten sus etapas de representación: enactiva, icónicas y simbólicas (Guilar, 2009). "A la primera etapa corresponde la formación de la inteligencia práctica, en donde la representación del mundo se realiza mediante la acción (respuesta motriz); la etapa icónica corresponde a la representación de las cosas a partir de imágenes, y la simbólica es cuando la acción y las imágenes se traducen en un lenguaje. En este sentido, lenguaje y desarrollo cognitivo son vinculantes y a su vez el lenguaje es instrumento de cognición" (Díaz, 1999: 222).

4 Durante las primeras décadas del siglo XIX la Escuela de los Annales, fundada por Marc Bloch y Lucien Febvre en 1929, cuestionó los planteamientos positivistas acerca de la historia, redefiniendo lo que hace científico al estudio histórico. Para lo cual se partió de reflexiones

Diólo@os 
En este marco, Dennis Shemilt realizó en Inglaterra el Proyecto 13-16 con el apoyo del School Council, organismo subvencionado por el Estado y encargado del desarrollo curricular en secundaria. El objetivo fue analizar el significado de la asignatura de historia en secundaria y bachillerato. Entre las conclusiones de esta investigación, se destacó que la historia debe enseñarse como una forma de conocimiento complejo y que solo a partir de la adolescencia los estudiantes poseen la madurez cognitiva para abordarlo. En las conclusiones se recomendó considerar la maduración cognitiva de los alumnos para establecer estrategias pertinentes, así como promover la empatía histórica de tal forma que ellos se identifiquen con los personajes y la época (Lamoneda, 1998)..$^{5}$

En los años setenta y ochenta hubo reformas en varios países, las cuales hicieron eco a los resultados y recomendaciones emanadas de las investigaciones. En España, por ejemplo, se vivió una profunda reforma educativa que implicó la modificación de planes y programas para el nivel básico y para la formación de profesores, la cual se elevó al nivel de estudios superiores y quedó a cargo de las universidades. Este hecho incidió en la creación de centros de investigación educativa.

En la Universidad de Valencia, por ejemplo, nació el grupo Germania 75, que cuestionó el enfoque positivista de enseñanza de la historia en la educación secundaria y propuso estrategias basadas en los conocimientos didácticos disponibles (Salles, 2011). En la Universidad de Barcelona, el acercamiento teórico se encaminó a las posturas psicogenéticas de la corriente inglesa. De este modo, se realizó una réplica del Proyecto 13-16, que se llamó Historia 13-16, con Prats al frente. La investigación se fundamentó en los avances de la psicopedagogía y se guio por el método por descubrimiento de Bruner, con el propósito de enseñar a los alumnos técnicas básicas de los historiadores que les permitieran una mejor comprensión de los conocimientos históricos y formar "ciudadanos críticos, libres y responsables" (Prats, 1989, citado en Salles, 2011). El proyecto planteaba también conocer cómo se aprende la historia, bajo el supuesto de que este proceso implica una forma específica de adquisición de conocimiento. Las conclusiones fueron similares a las del proyecto inglés: que en la adolescencia es posible tanto la comprensión de la metodología histórica por parte de los alumnos como la aplicación de algunos aspectos del trabajo del historiador dentro del aula (Fairstein, Carretero, 2001; Salles, 2011). Además de la influencia inglesa en el Proyecto Historia 13-16, otro punto clave para el caso español fue la historiografía francesa, cuyos referentes sirvieron de base al poner menos énfasis en los contenidos y promover, mediante acercamientos continuos, el aprender la investigación histórica.

en torno al quehacer del historiador: ¿Qué significa, desde la historia, pensar históricamente? y ¿cómo se llega a dicho pensamiento? El pensar históricamente se relacionó con un oficio, la construcción de la historia a partir de la metodología propia de dicha disciplina. Estas ideas sirvieron de base para que, décadas después, se buscara emular el trabajo del historiador en las propuestas de enseñanza.

5 Al respecto, Plá (2014: 257) señala que la idea de la empatía histórica fue cuestionada a inicios del siglo XXI, aduciendo que conduce al anacronismo en lugar de contribuir a pensar históricamente, ya que deja de lado el contexto histórico de quien analiza y "[no considera] los aspectos imperceptibles para los actores del acontecimiento histórico que [solo] pueden observarse con la distancia temporal". 
Con lo expresado hasta aquí, podemos inferir que la investigación en enseñanza y aprendizaje de la historia logró cierto grado de institucionalización en las universidades, lo que se observa en el desarrollo de proyectos en centros especializados e investigadores abocados a esta tarea. En España, las investigaciones de los años setenta y ochenta dieron paso a las didácticas específicas, quedando la historia incluida en la didáctica de las ciencias sociales. Además, en las reformas curriculares de la década de los noventa, en el marco de la Ley de Ordenación General del Sistema Educativo, las didácticas específicas fueron parte de los planes y programas de estudio para la formación del profesorado y estuvieron presentes en las propuestas para la educación básica (Pagès, 1994, 2012; Prats, Valls, 2011).

¿Qué ocurrió en México? Aquí fueron los y las historiadoras quienes se acercaron a la investigación en enseñanza de la historia, participaron en las reformas curriculares y en la elaboración de los libros de texto. En los años setenta, un importante grupo de académicos/as de El Colegio de México apoyó en la elaboración de los nuevos libros de texto de historia para la educación primaria, ampliando los contenidos más allá de la historia política y articulándolos en una lógica de complejidad ascendente. En nuestro país, no debemos soslayar la importancia de los libros de texto. Su carácter único, gratuito y obligatorio en todas las escuelas, los convierten en un poderoso instrumento de irradiación de conocimientos $y$, a la vez, suelen ser el material central de la enseñanza y del aprendizaje, sobre todo en contextos donde el acceso a la cultura escrita está mediado casi exclusivamente por la escuela.

A pesar de los cambios en los libros y en los contenidos, las bases pedagógicas de los planes y programas de estudio poco tenían que ver con las aportaciones de la pedagogía genética y cognitivista, y estaba prácticamente ausente el emular el trabajo de los historiadores. Los programas de educación primaria y secundaria fueron organizados con base en la tecnología educativa, de filiación fundamentalmente conductista. Además, en la formación y en la práctica docente se siguieron utilizando formas tradicionales para la enseñanza de la historia: exposición del maestro, elaboración de resúmenes y resolución de cuestionarios.

En síntesis, podemos decir que en esta primera etapa, en Estados Unidos y en Europa la investigación en enseñanza de la historia atendió tres interrogantes: ¿Cómo se forman las nociones temporales? ¿cuál es la etapa en que mejor se aprende el conocimiento histórico? ¿es posible acelerar la adquisición del conocimiento histórico? ¿cómo? El conocimiento producido incidió en la creación de nuevos diseños curriculares y materiales educativos orientados a fomentar una enseñanza basada en contenidos más de corte social y económico y menos político, en intentar dejar de lado la memorización de fechas para tratar de comprender los procesos históricos de forma integradora; además de plantear el uso del método de investigación del historiador. En México se hicieron algunos intentos, sobre todo por trasformar los contenidos escolares y por ensayar algunas innovaciones didácticas.

Diálo@os sobre Educación 
A pesar de las investigaciones, las reformas, las sugerencias didácticas y el diseño de nuevos libros, las propuestas tuvieron poco éxito en las aulas y se mantuvo una enseñanza de tipo tradicional basada en el contenido y en la memorización, mostrando con ello desfases entre lo que la academia señalaba y lo que sucedía en la práctica real. Los diferentes contextos escolares influyeron en la aplicación de las innovaciones, siendo los actores escolares (profesores, alumnos, directivos) y las comunidades los principales opositores. De ahí que en los años noventa, algunas de las preocupaciones de los investigadores giraron en torno a: ¿Por qué fracasan las reformas? ¿qué pasa con los profesores? ¿de qué forma los padres, los administradores escolares y los contextos limitan la operación de las reformas? ¿es posible formular una didáctica específica para la enseñanza de determinada disciplina?

\section{La didáctica de la historia (1990-2000)}

Desde finales de los ochenta la investigación en enseñanza de la historia experimentó algunos desplazamientos en los problemas y objetos de interés, mismos que cobraron mayor fuerza en los años siguientes, y se enmarcan en tendencias más amplias en las que están implicadas la psicología educativa y la didáctica. Como ya señalamos, uno de estos desplazamientos giró en torno a explicar por qué fracasan las reformas, integrando a este cuestionamiento la preocupación por entender el papel del profesor como mediador de los cambios curriculares y cómo actúan sus marcos de referencia en la enseñanza. El segundo desplazamiento partió de pensar en el currículo como una construcción histórica y social, lo que llevó a reflexionar sobre su dimensión normativa y operativa desde las didácticas específicas (Pagès, 1994; Prats, Valls, 2011 ).

En este contexto, en Estados Unidos las investigaciones enfatizaron el papel de los actores locales, en tanto agentes que median, resisten, negocian o se oponen a las reformas; esto, con el ánimo de entender por qué las reformas y las innovaciones pedagógicas basadas en el cognitivismo estaban poco presente en las aulas. Al respecto, Casanova y Berliner (1997) indican que el cognitivismo tuvo una fuerte incidencia en la investigación educativa enfocada en los procesos de formación del conocimiento; sin embargo, en las escuelas su penetración fue débil. Por ejemplo, el estudio de Barry Franklyn (1991) mostró que a nivel local las autoridades, los profesores y los padres de familia obstaculizaban las trasformaciones educativas referidas a la enseñanza de la historia y a las ciencias sociales. Este mismo autor cita el estudio pionero de McKinney y Westbury (1975) acerca de tres intentos de reforma en la ciudad de Gary, en Indiana, entre 1940 y 1970, los que tuvieron un impacto leve o de corta duración debido al escaso apoyo de los actores locales, el poco financiamiento y la falta de materiales e infraestructura.

Una segunda línea de investigación consistió en problematizar sobre los aspectos culturales implicados en la enseñanza de la historia, en relación con la formación de las nociones temporales y el desarrollo. La reflexión teórica llevó a considerar un concepto clave planteado por Vigotsky, las funciones psicológicas superiores, lo que permitió debatir sobre los aspec- 
tos histórico-culturales, el lenguaje y la interacción social como mediadores de los procesos de aprendizaje. Con ello se trasformó la visión del desarrollo evolutivo formulado por Piaget hacia una visión del desarrollo como un proceso inserto en dinámicas sociales. Las ideas de Vigotsky (1979) penetraron en las diferentes disciplinas que hasta el momento se relacionaban de forma un tanto difusa en la investigación en enseñanza de la historia: la psicología educativa, la historia y la didáctica. ${ }^{6}$

En España, esos desplazamientos se hicieron presentes en los trabajos de Prats y Valls (2011), ambos adscritos a la Universidad de Barcelona. A mediados de la década de los ochenta, ellos participaron en los cuestionamientos a la postura psicogenética y subrayaron la importancia de la cultura y el contexto del alumno en la enseñanza y aprendizaje de la historia. Dichas críticas partieron de la revisión de las posturas evolutivas en comparación con lo que mostraban los estudios dentro del aula. Fue entonces que se buscó fundamentar la didáctica de la historia con los avances propuestos por las teorías de corte sociocultural, perspectiva teórica de la que se derivaron varios postulados. El primero se refería a que la enseñanza de la historia implica no solo el contenido de la materia, sino un saber hacer, pues "diferentes materiales, diferentes contextos y situaciones demandan diferentes tipos de pensamiento" (Booth, 1987, citado en Carretero, Limón, 1993: 157). Un segundo postulado consistía en afirmar que el conocimiento histórico es un tipo de pensamiento específico, con "su propia lógica, métodos y perspectivas" (Shemilt, 1987, citado en Carretero, Limón, 1993: 155). En tercer lugar, se planteó la importancia de relacionar la reflexión teórica con las evidencias de lo que ocurría en el aula (Carretero, Limón, 1993: 155).

Con base en esos postulados, hubo nuevas preguntas: ¿Qué tipo específico de conocimiento es el conocimiento histórico? ¿cómo enseñarlo? Para encontrar respuestas, los académicos construyeron nuevas líneas investigación insertas en las didácticas específicas en ciencias sociales e historia. ${ }^{7}$ El foco de interés consistía en cómo construir mediaciones entre el conocimiento disciplinar y el alumno, es decir, cómo "escolarizar" el conocimiento histórico considerando los contextos y el desarrollo psicológico de los alumnos, para lo cual era necesario diseñar estrategias que lo hicieran comprensible y aprehensible.

6 Para Condliffe (2003), en Estados Unidos los campos que estudiaban la educación empezaron a separarse desde la década de los setenta. Las diferentes disciplinas que estudian la educación se aislaron unas de otras. Surgieron subespecialidades en ciencias sociales enfocadas en estudios educativos formando dos grupos, uno basado en las disciplinas (historia de la educación, filosofía de la educación, psicología de la educación, etc.), y otro centrado en la función social de la educación. Para la autora, el primer enfoque fue el que dio mayores resultados pues se desarrollaron investigaciones críticas desde distintas disciplinas que acercaron a historiadores al campo educativo.

7 Para el caso de España, Prats y Valls (2011) plantean que durante los ochenta comenzaron a surgir en las universidades asignaturas enfocadas en didáctica de las ciencias sociales. Sin embargo, es hasta el año 2000 que creció el interés de historiadores por el área de la Didáctica de la historia, lo cual se reflejó en la apertura en la carrera de Historia de áreas enfocadas en la enseñanza y didáctica, y también en el aumento de tesis doctorales. Esto ocurrió principalmente en la Universidad de Barcelona. Para el caso de México, a finales del siglo XX sucede algo similar, Victoria Lerner (citada en Latapí, 2014), en sus publicaciones de finales de los noventa planteó la importancia de incluir en la carrera de Historia asignaturas sobre didáctica de la historia, también señaló que era necesario conformar un campo de investigación en la enseñanza de la historia que alimentara a la didáctica con propuestas.

Diálo@os 
Estas preocupaciones también fueron tratadas por algunos historiadores. Tal es el caso de Jacques Le Goff y Antonio Santoni (1996), quienes en un texto escrito por ambos y que circuló ampliamente en México, invitaban al diálogo entre historiadores, pedagogos e interesados en la enseñanza de la historia, para pensar en la importancia cultural y formativa de la disciplina con el propósito de formar un programa y un lenguaje en común. Para ello, indicaron que era necesario que los jóvenes comprendieran cómo se construye la historia, imitando los objetivos, preguntas, métodos y recursos utilizados por los historiadores. El docente, por su parte, debería conocer los planteamientos historiográficos.

En México, estas ideas y debates también tuvieron eco. Lo cual se puede observar, por ejemplo, en el Congreso promovido por Victoria Lerner y celebrado en la Universidad Nacional Autónoma de México en 1988. Los trabajos presentados se organizaron en tres bloques temáticos: a) Prácticas de la enseñanza de la historia, en donde se presentaron experiencias, b) Didáctica de la historia, que concentró las propuestas, y c) Reflexiones teóricas, que incluyó ponencias acerca de la relación entre la enseñanza de esta disciplina y otras ciencias sociales (Lerner, 1990).

Este esfuerzo tuvo continuidad, principalmente, a través de un destacado grupo de historiadoras. Victoria Lerner (1990), Mireya Lamoneda (1998), Luz Elena Galván (2006) y Andrea Sánchez (2006) denunciaron que en las escuelas persistían formas tradicionales de enseñanza, y señalaron que era necesario escolarizar el trabajo del historiador y del conocimiento histórico; también incorporaron en sus preocupaciones los debates pedagógicos producidos en España y elaboraron propuestas didácticas.

Estas académicas enfatizaron que la enseñanza debería considerar el contexto de los alumnos como una forma de acercamiento a las posturas cognoscitivas. En particular, Lamoneda (1998) indicó que teóricamente se asumía que la adolescencia era la etapa de consolidación del pensamiento abstracto y del tránsito hacia formas de pensamiento más maduras, como lo es comprender la complejidad del tiempo y el espacio, pero la experiencia docente mostraba que los alumnos difícilmente se apropiaban de dichos conceptos. Para ella, este problema se debía a la ausencia de contenidos y estrategias que permitieran relacionar lo aprendido con el entorno, por lo que proponía el uso de mediadores específicos, con la finalidad de formar un puente entre el conocimiento histórico y los alumnos. Dichos mediadores debían considerar que la historia es un conocimiento con naturaleza propia de tipo formal, que requiere ciertas especificidades para ser enseñado y aprendido.

Historiadores mexicanos como Luis González y González (1998) e Hira de Gortari (1998), más cercanos al trabajo de la investigación histórica que a los fundamentos psicológicos y didácticos para su enseñanza, propusieron partir de preguntas problema y utilizar fuentes históricas en los salones de clase; todo con la finalidad de acercar al alumno al método del historiador y generar una enseñanza crítica. Ambos cuestionaron la enseñanza de la historia, sin embargo, 
sus ideas partían de problemáticas que observan en la práctica educativa y de su experiencia como historiadores, pero sin que existiera una investigación sistemática que fundamentara sus ideas.

Para finales de los noventa, en México, la investigación educativa estaba en expansión y la investigación sobre las didácticas específicas era visible. Así se observó en el Consejo Mexicano de Investigación Educativa (COMIE), concretamente en el campo de la enseñanza de la historia, Luz Elena Galván participó en la hechura de los primeros estados del conocimiento que abarcan la década de los ochenta y las perspectivas para los noventa. ${ }^{8}$ Paulina Latapí (2014) señala que en México, a partir de los trabajos elaborados en ese entonces, se vislumbró con mayor claridad la importancia de la delimitación del campo de enseñanza de la historia, su conceptualización teórica y metodológica, así como la necesidad de avanzar en trabajos formales de investigación.

En cuanto a las reformas curriculares llevadas a cabo en México en los años ochenta y noventa, en estas se cuestionó la tecnología educativa y se difundió la didáctica de la historia y de las ciencias sociales con la intención de llevar el conocimiento disciplinar a las aulas. También se buscó difundir el método de la historia y conceptos como la secuenciación temporal, las relaciones causa y efecto, las ubicaciones espaciales, el planteamiento de preguntas y el uso de fuentes. Con base en lo anterior, se modificaron los planes y programas de educación básica, los libros de texto y materiales de apoyo para el maestro, así como la formación en las escuelas normales. Si bien estos desplazamientos tuvieron eco en los diseños curriculares, son pocos los estudios que indaguen acerca de lo que ocurrió en la práctica; tampoco hay investigaciones sobre cómo se formaron los docentes de historia en esas décadas. Al respecto, Alanís y Medina (2019: 99) subrayan que "La investigación y las innovaciones pedagógicas han circulado en los medios académicos a un ritmo, mientras que su adopción en experiencias escolares concretas ha sido muy limitada".

Los debates suscitados en el periodo analizado en este apartado condujeron a replantear la investigación en enseñanza de la historia, colocando a la didáctica en un lugar preponderante. De este modo, el reconocimiento sobre la importancia de los aspectos socioculturales y de mediadores, en especial del lenguaje como herramienta fundamental para los procesos de enseñanza-aprendizaje, justificaron la creación de las didácticas específicas centradas en diseñar puentes entre el conocimiento disciplinar y su enseñanza y aprendizaje. El supuesto que sostiene esta posición es que "el habla se reconoce como un instrumento, un intermediario para el pensamiento superior" (Patiño, 2007: 55). Asimismo, al iniciar el siglo XXI se dieron las condiciones para buscar una integración entre la psicología educativa, la metodología propia

8 El interés por las didácticas específicas, que articularan el conocimiento disciplinar con las teorías pedagógicas y la enseñanza, permeó el trabajo al interior del COMIE. En 1996 se publicó el texto coordinado por Waldegg y Block, Estudios en didáctica, que incluía varios trabajos sobre enseñanza y aprendizaje de las ciencias sociales.

Diólo fos 
del historiador y la didáctica de la enseñanza de la historia. Proceso que implicó una restructuración tanto teórica como metodológica del campo de investigación en enseñanza de la historia, la cual aún está en proceso.

\section{México: debates y aportaciones recientes}

Como consecuencia de los cambios económicos, sociales y políticos impulsados por el fin de la guerra fría, entre los últimos años del siglo XX y primeros del XXI se trasformó la manera de comprender el mundo. Carlos Díaz (1999) indica que el conocimiento y la tecnología se convirtieron en piedras de toque de la "sociedad del conocimiento", en tanto motores de producción económica y de generación de riqueza. En este contexto, la educación fue vista como un mecanismo relevante para generar un capital humano que atendiera los nuevos retos de producción. Lo anterior debido a que, ante la rapidez de los cambios, el conocimiento pormenorizado y específico pierde relevancia, por lo que el énfasis se pone en el papel de la educación para lograr el desarrollo de habilidades y destrezas flexibles que permitieran al individuo estar preparado para la sociedad del conocimiento, al contar con disposición permanente para el aprendizaje. El problema era ¿cómo lograrlo? ${ }^{9}$

En este contexto, los debates que circularon en torno a la enseñanza de la historia experimentaron algunos desplazamientos. Un primer conjunto de cuestionamientos se vincula con la tradición de las didácticas específicas y con preocupaciones prácticas: ¿Cuáles serían las habilidades necesarias para lograr un pensamiento histórico perdurable? ¿qué mediaciones o estrategias serían necesarias? ¿qué deberían saber y hacer los profesores para lograr el aprendizaje de la historia? Una segunda vertiente de interés plantea la necesidad de elaborar investigaciones sistemáticas, rigurosas y profundas, lo que lleva a postular la complejidad del objeto de estudio llamado enseñanza de la historia y a proponer enfoques teóricos y metodológicos inter y transdisciplinares.

Estas dos tendencias se pueden nombrar, siguiendo a Plá $(2005,2008,2012)$, como educación histórica e investigación en enseñanza de la historia. La primera enfatiza la investigación pedagógica y didáctica, privilegia la investigación aplicada, ensaya propuestas, analiza resultados, propone métodos, estrategias y medios de enseñanza y aprendizaje para la formación del pensamiento histórico. Por otro lado, la investigación en enseñanza de la historia está más interesada en comprender los distintos procesos y realidades de este objeto de estudio, así como sus significados, por lo que enfatiza el papel de los actores, sus producciones y el contexto.

El hecho de que Plá proponga estos ámbitos de investigación se da en el marco de un creciente interés por la enseñanza de la historia; mismo que se observa en el aumento de tesis

$9 \mathrm{El}$ constructivismo es un enfoque integrador, en tanto su objetivo es: "configurar un esquema de conjunto orientado [a] analizar, explicar y comprender los procesos escolares de enseñanza aprendizaje" (Coll, 1997, citado en Fairstein, Carretero, 2001: 180). De manera similar, Díaz (1999) señaló que el constructivismo es una serie de postulados anclados en distintas psicologías del desarrollo y del aprendizaje como la psicología genética, el cognitivismo, el aprendizaje significativo y la corriente sociocultural. 
sobre el tema, ${ }^{10}$ en las trasformaciones curriculares para la formación docente y en la aparición y fortalecimiento de redes de investigación. Sobre este último punto, Latapí (2014) señala que en 2000 se formó la Asociación Mexicana de Investigación en Didáctica de la Historia (AMIEDH), que buscaba vincular la investigación con la práctica docente. ${ }^{11}$ Por su parte, la Red Nacional de Especialistas en Docencia, Difusión e Investigación en Enseñanza de la Historia (REDDIEH) nació a partir de los coloquios en enseñanza de la historia, organizados en 2006 por Hugo Torres, y en estrecha relación con la Red Nacional de Licenciaturas en Historia y sus Cuerpos Académicos (RENALIHCA).

La conformación de estos colectivos propició un espacio de encuentro en donde es posible observar la heterogeneidad de ideas, preocupaciones y planteamientos; el entrecruzamiento de temáticas, el debate académico y la integración de algunas posturas. Los ocho encuentros realizados por la REDDIEH son un punto de referencia de lo que ocurre en distintas instituciones de educación superior relacionadas con la docencia de la historia. En la actualidad, incluye no solo a los especialistas de México, sino también de otros países, como España, Colombia, Perú, Argentina, Brasil, Chile y Cuba. ${ }^{12}$ Entre las temáticas tratadas, hay un número importante de investigaciones sobre la práctica docente y acercamientos novedosos, por ejemplo, en torno a la teoría de las representaciones sociales y las creencias de los profesores. En la última década se han incrementado los trabajos que podrían entrar en las áreas de investigación propuestas por Plá, la educación histórica y la investigación en enseñanza de la historia. De ahí que enseguida revisemos estas dos líneas de trabajo.

\section{La educación histórica}

Ha sido ampliamente desarrollada en Inglaterra y en España. Dentro de las preocupaciones de los especialistas adscritos a esta área de conocimientos están cuestiones como: ¿para qué enseñar la historia? ¿cómo debe ser enseñada? y ¿qué procesos están imbricados en su aprendizaje? ${ }^{13}$

10 Según datos aportados por Latapí (2014), de 2000 a 2010, la producción mejor fundamentada en el campo de la investigación en enseñanza de la historia estaba en las tesis. Ella encontró 41 trabajos en esa década: de maestría 58\%, de licenciatura 38\% y de doctorado 4\%. Los temas más recurrentes fueron propuestas pedagógicas y didácticas con $44 \%$, análisis sobre el estado de la enseñanza 17\%, reformas, políticas, planes y programas de estudio $17 \%$, libros de texto $12 \%$, identidad y educación indígena $5 \%$, e inclusión de la perspectiva de género en la enseñanza de historia 5 por ciento.

11 En el marco de los Estados de Conocimiento producidos en el Consejo Mexicano de Investigación Educativa, esta asociación fue la responsable de elaborar el de "Didáctica de las ciencias histórico-sociales" de 1993 a 2002.

12 De España, cabe destacar la presencia de Joan Pagès en la mayoría de esos encuentros, con valiosas aportaciones. La comunidad de investigadores lamenta su muerte, ocurrida el 3 de junio de 2020.

13 En 2011 la Secretaría de Educación Pública editó un interesante material destinado a los docentes, titulado Enseñanza y aprendizaje de la historia en educación básica, en el cual ya se incluyen algunos elementos de la educación histórica, aunque en tensión permanente con la abundancia de contenidos definidos por el Estado. En este material participaron como autores Joaquín Prats / Universidad de Barcelona, España; Juan Santacana / Universidad de Barcelona, España; Laura H. Lima Muñiz y Ma. del Carmen Acevedo Arcos / Universidad Pedagógica Nacional-Ajusco, México; Mario Carretero Rodríguez / Universidad Autónoma de Madrid y FLACSO-Argentina; Pedro Miralles Martínez / Universidad de Murcia, España; y Verónica Arista Trejo / Maestra de Educación Secundaria, México. 
Algunos de sus conceptos centrales son el pensar históricamente, el pensamiento histórico, el tiempo histórico y la conciencia histórica. ${ }^{14}$

En España, Pagès ha estudiado con detenimiento el pensamiento histórico y el desarrollo de la conciencia histórica y temporal. Por su parte, Carretero y Limón (1993), y Carretero y Rodríguez (2009) han definido los conceptos de primer y segundo orden, que sirven para comprender cómo se da la estructuración secuenciada del pensamiento histórico en los aprendices y para diseñar propuestas didácticas. En México, sus aportaciones tuvieron resonancia y sirvieron de base para reflexionar sobre las implicaciones cognitivas de la enseñanza de la historia, de tal modo que, al usar los aportes de la psicología para comprender el desarrollo del pensamiento histórico, lo que se hizo fue "desplazar significados de la psicología a significantes de corte historiográfico, hasta convertirlos en conocimiento histórico escolar" (Plá, 2012: 270). Sobre estos postulados básicos, en los últimos 20 años se diseñaron nuevos planes de estudio para la enseñanza de la historia, tanto en educación básica como para la formación de profesores.

Sebastián Plá (2012) considera que en la primera década del siglo XXI se trasformó el objetivo escolar tradicional de la historia, el cual ayudaba a configurar una identidad nacional para plantearse una visión instrumental de la historia. Así, la respuesta a la pregunta, Historia ¿para qué?, deja de ser la construcción de la conciencia nacional y se promueve la generación de habilidades específicas. Ahora se le confiere una importancia nodal a la formación de habilidades del pensamiento. ${ }^{15} \mathrm{El}$ contenido histórico pasa a un segundo término ya que se privilegia el enseñar a pensar y a hacer historia, se aprende historia para generar habilidades relacionadas con la búsqueda y crítica de información, y para formar una ciudadanía capaz de articular lo local con lo global.

Es en este contexto que Arteaga y Camargo (2014) reconocen que la enseñanza de la historia ha pasado por tres estadios, uno en el que lo central eran los contenidos, otro que buscó discernir cómo lograr convertir el saber especializado en saber aprendido y, el último, que busca conocer cómo se aprende a pensar históricamente, y a partir de ese conocimiento, generar propuestas. El problema que plantea la educación histórica es entonces cómo dejar atrás la centralidad de abundantes contenidos, ordenados en rigurosa cronología y con criterios de excesiva uniformidad, para construir aprendizajes que, desde la diversidad, posibi-

14 La conciencia histórica se define como la comprensión de la relación entre el pasado, el presente y el futuro, y conlleva discernir sobre la temporalidad, sus movimientos y articulaciones (Santiesteban, 2017).

15 Según Gallardo y Novillo, al relacionar el aprendizaje con el desarrollo del pensamiento en la enseñanza de la historia habría que pensar en tres tipos de pensamiento en los alumnos: crítico, creativo e histórico. Su idea es alentar la práctica constante de la crítica, motivando la reflexión de una situación con el trabajo directo con fuentes, para enfrentar al alumno a las varias interpretaciones de un mismo hecho: "saber trabajar con interpretaciones en lugar de certezas implica desarrollar un pensamiento crítico" (2017: 162). Para estos autores, el pensamiento histórico implica la formación de una conciencia temporal que permita relacionar el presente con el pasado y el futuro, y crear así una conciencia histórica. Estas aseveraciones parten del Proyecto Zero, puesto en marcha en la Universidad de Harvard desde la década de los setenta y que continua en la actualidad, su objetivo es realizar investigación acerca de "la educación como comprensión e impulso del pensamiento" (2017: 157). 
liten la apropiación de un pensamiento histórico y de una conciencia crítica, en el sentido de reconocer(nos) como seres sociales configurados por diversos estratos del pasado.

La propuesta es hacer una historia situada, una historia en contexto. De ahí que "la tarea de la educación histórica es promover la comprensión cada vez más profunda sobre la naturaleza de la disciplina, al mismo tiempo que se aprende sobre el pasado y las huellas de este en la vida actual" (Arteaga, Camargo, 2014: 122). Para alcanzar ese pensamiento histórico, para aprender a pensar históricamente, es necesario considerar una enseñanza y un aprendizaje en espiral, por etapas, que va escalando niveles de complejidad mediante el desarrollo de conceptos ordenadores, y que implica ubicar los contextos, los puntos de partida y las metas, considerando las tareas de quien enseña y de quien aprende.

Según Plá (2012), la preocupación central de la educación histórica es analizar y proponer el tipo de habilidades que se requiere formar, para lo cual se vinculan: a) la psicología educativa, a partir de las propuestas del cognitivismo, b) la didáctica, mediante procedimientos y materiales de enseñanza-aprendizaje acordes al conocimiento histórico que se busca construir, y c) la historia, al retomar parte de sus métodos y escolarizarlos mediante estrategias didácticas operables en la práctica educativa.

\section{Investigación en enseñanza de la historia}

Ahora bien ¿Cuál es la propuesta de la investigación en enseñanza de la historia? A diferencia de las investigaciones ubicadas en el campo de la educación histórica, que indagan y proponen acerca de las habilidades relacionadas con el conocimiento histórico y con el desarrollo del pensamiento histórico, la investigación sobre enseñanza de la historia retoma dos preguntas de viejo cuño, ¿qué historia? y ¿para qué?, pero las revitaliza en el horizonte de las discusiones de las ciencias sociales.

Para abordar esta problemática, Plá (2012) propone analizar las relaciones entre discursos, significados y prácticas, buscando comprender cómo se enlazan en la escuela presente y pasado, y cómo, desde ese punto de encuentro, se perfilan escenarios futuros. En esta perspectiva tienen cabida diferentes versiones del pasado, las cuales se imbrican en situaciones de tensión, inconsistencia, coexistencia o hegemonía. El reto es incluir tanto los discursos elaborados por las elites como la perspectiva de los actores escolares, con el objetivo de comprender los procesos de resignificación, apropiación, simulación o resistencia, entre otros posibles.

Se trata de una propuesta que focaliza la escuela como el lugar de la indagación y a los sujetos que significan, de forma inter e intrasubjetiva y desde su presente, versiones distintas del pasado, aceptando que no necesariamente reproducen el discurso de la historia o de los reformadores. De este modo, la perspectiva de investigación en enseñanza de la historia reconoce que las personas están en un lugar sociocultural desde el cual se relacionan con los 
conocimientos de la historia formulados por los especialistas (Plá, 2008, 2012). Lo anterior no significa negar la existencia de un discurso marco, pero el interés es leer su presencia o ausencia y su entrecruzamiento con lo que ocurre en la "caja negra de la escuela".

En ese sentido, la investigación en enseñanza de la historia es propicia para reflexionar sobre cómo es que en determinada época y contexto se produce un modelo de historia por enseñar y cuál es la historia que realmente se enseña. De ahí que se elaboren preguntas sobre las ideas, concepciones e intenciones, pero también se ocupa de escudriñar qué pasa en las prácticas educativas y en el pensamiento de alumnos y profesores. Sobre lo anterior, Sebastián Plá (2012) señala que es importante que se conciba la enseñanza de la historia como objeto de investigación y aboga por construir un campo autónomo que integre distintas disciplinas. Para lo cual sería necesario entender el porqué de las reformas y ubicarlas en su momento histórico; hacer investigaciones en los contextos específicos para comprender las prácticas y los sentidos de la historia que son elaborados por los sujetos. Lo cual requiere, a su vez, pensar con el utillaje teórico y metodológico, no solo de la pedagogía, la psicología educativa o la didáctica, sino de las ciencias sociales.

Ahora bien, el que educación histórica e investigación en enseñanza de la historia se presenten como dos líneas de investigación, no quiere decir que tengan diferencias profundas o que sea imposible plantear objetos de estudio montados entre ambas. En una lógica de integración, diálogo y debate, Ana Zavala (2015), por ejemplo, coincide con Plá en ubicar la mirada en la escuela y en el aula para entender cómo se enseña y se piensa la historia. Sin embargo, Zavala se enfoca en la práctica y en el profesor; en cambio, Plá se ha interesado por las tramas discursivas de los sujetos. Ambos autores destacan lo importante de considerar la voz de los sujetos para acceder a las experiencias y objetivar la subjetividad. Este posicionamiento implica asumir que las personas están cruzadas por diferentes sentidos y significados, pues lo que se busca es conocer cómo piensan y practican la enseñanza y el aprendizaje de la historia, y explicar por qué. De tal posicionamiento se derivan decisiones metodológicas cercanas a los enfoques cualitativos, lo que lleva a dialogar, de forma profunda y sostenida, con las ciencias sociales, para poder discernir cómo se relacionan o tensan lo individual con lo colectivo y lo local, con lo nacional y lo global.

\section{Consideraciones finales}

Resolver la pregunta ¿cómo se configuró el campo de conocimiento sobre la enseñanza y aprendizaje de la historia?, nos llevó a trazar un territorio en el que ubicamos algunos de los participantes en esa travesía y retomamos sus recorridos para conocer sus ideas y propuestas. Como todo trazo, nuestro trabajo es apenas un bosquejo, por lo que quedan pendientes imágenes y líneas por definir en torno, por ejemplo, a qué huellas dejaron en las aulas de las escuela los diversos intentos de reforma y cómo fueron recibidas por los maestros, qué particularidades 
tiene el conocimiento histórico escolar con respecto a otras ciencias sociales, cómo se dan -en contexto específicos- los procesos de apropiación de los contenidos conceptuales, procedimentales y actitudinales propuestos en el marco de la educación histórica. También consideramos necesario avanzar en investigaciones sistemáticas y bien fundamentadas que impliquen el diseño, puesta en práctica y evaluación de propuestas didácticas. A pesar de estos pendientes, consideramos que lo expuesto en este documento ayuda a dilucidar algunos de los principales recorridos y desplazamientos implicados en el proceso de configuración del campo de investigación en la enseñanza y aprendizaje de la historia.

Otro tema pendiente es incrementar el diálogo entre historiadores, expertos en la investigación en enseñanza de la historia y profesores. Pensamos que es hora de promover investigaciones con carácter más horizontal y colaborativo para avanzar en un enriquecimiento mutuo y dilucidar, por ejemplo, ¿qué pasa en la enseñanza y el aprendizaje de la historia en distintos contexto y niveles? ¿cómo se apropian los docentes y los estudiantes de los conceptos de primer y segundo orden? ¿es posible enseñar y aprender una conciencia histórica? ¿cómo se construye esa conciencia histórica? ¿qué papel tienen los contextos, los sujetos, las culturas institucionales en esa construcción? También queda mucho por hacer y por entender en relación con el uso de las tecnologías de la información y la comunicación en la gestión de la educación histórica, o bien, en los efectos de estas tecnologías en la fragmentación o pérdida de sentido de las historias individuales y locales, y los efectos de estos procesos en la construcción de identidades colectivas. En cualquier caso, la apuesta está en que aprender a pensar históricamente contribuye a generar en los individuos una conciencia crítica y da herramientas para la reflexión, para lo cual es necesario que el campo de la investigación de enseñanza de la historia continúe fortaleciendo y promoviendo los debates inter y transdisciplinares.

\section{Bibliografia y referencias}

Alanís, F.; R. Medina (2019). El oficio de enseñar. Una experiencia docente en posgrado en historia. San Luis Potosí: El Colegio de San Luis.

Arteaga, B.; S. Camargo (2014). Educación histórica: una propuesta para el desarrollo del pensamiento histórico en el plan de estudios de 2012 para la formación de maestros de Educación Básica. Revista Tempo e Argumento, 6(13), 110-139. Florianópolis: UDESC.

Booth, M. (1987). Ages and Concepts: A Critique of the Piagetian Approach to History Teaching. En Portal, Ch. (ed.). The History Curriculum for Teacher. Londres: The Falmer Press.

Bruner, J. (1963). El proceso de la educación. México: UTEHA.

Diólo pos año 12 | número 22 | enero-junio 2021 | ISSN 2007-2171 
Carretero, M.; M. Limón (1993). Aportaciones de la psicología cognitiva y de la instrucción a la enseñanza de la historia y las ciencias sociales. Infancia y Aprendizaje, (62-63), 153-167. https://dialnet.unirioja.es/servlet/articulo?codigo $=48433$

Carretero, M.; C. Rodríguez (2009). Estudios cognitivos sobre el conocimiento histórico: aportaciones para la enseñanza y alfabetización histórica. Enseñanza de las Ciencias Sociales, (8), 75-89. https://www.raco.cat/index.php/EnsenanzaCS/article/view/184396/237469

Casanova, Ú.; D. Berlinder (1997). La investigación educativa en Estados Unidos: el último cuarto de siglo. Revista de Educación, (312), 43-80. http://www.educacionyfp.gob.es/dam/ jcr:3b0e44ae-8b70-48d6-900d-63adaed8b862/re3120400460-pdf.pdf

Coll, C. (1997). Constructivismo y educación escolar: ni hablamos siempre de lo mismo ni lo hacemos siempre desde la misma perspectiva epistemológica. En Rodrigo, M. J.; J. Arnay (comps.). La construcción del conocimiento escolar. Barcelona: Paidós, 107-136.

Condliffe, E. (2003). La investigación educativa en Estados Unidos: reflexiones para la historia. Revista Mexicana de Investigación Educativa, 8(18), 547-560. http://www.redalyc.org/articulo.oa?id=14001810

De Gortari, H. (1998). El reto de enseñar historia. Entrevista con Hira de Gortari Rabiela. Cero en Conducta, (46), 13-24. https://www.ceroenconducta.org/revistas/Revista46/Revista46Sello \%28arrastrado\%292.pdf

Díaz, C. (1999). Viejas y nuevas ideas en educación. Una historia de la pedagogía. Madrid: Popular.

Duckworth, E. (1981). O se lo enseñamos demasiado pronto y no pueden aprenderlo o demasiado tarde y ya lo conocen: el dilema de «aplicar a Piaget». Infancia y Aprendizaje: Journal for the Study of Education and Development, (2), 163-177. http://antigositebolsa.fde.sp.gov. br/Eleanor Duckworth.pdf

Fairsten, G.; M. Carretero (2001). La teoría de Jean Piaget y la educación. Medio siglo de debates y aplicaciones. En Trilla, J. (coord.). El legado pedagógico del siglo XX para la escuela del siglo XXI. Barcelona: Graó, 177-207.

Franklyn, B. (1991). La historia del currículum en Estado Unidos, status y agenda de investigación. Revista de Educación, (295), 39-57. https://www.mecd.gob.es/dctm/revista-de-educacion/articulosre295/re29502.pdf?documentld=0901e72b813577e5

Galván, L. E. (2006). Teoría y práctica en la enseñanza de Clío. En Galván, L. E. (coord.). La formación de una conciencia histórica. México: Academia Mexicana de la Historia, 219-241.

Gallardo, J.; M. A. Novillo (2017). Aprender historia con rutinas de pensamiento. Proyecto Clío, (43), 153-179. http://clio.rediris.es/n43/articulos/GallardoNovillo2017.pdf

González, L. (1998). ¿Qué historia enseñar? Entrevista con Luis González y González. Cero en Conducta (46), 5-11. https://www.ceroenconducta.org/revistas/Revista46/Revista46Sello \%28arrastrado\%29 1.pdf 
Granja, J. (2000). Configuración de conocimientos sobre la educación. Colección Pedagógica Universitaria, (32-33), 55-71. https://www.uv.mx/cpue/colped/N 32 33/Configuracion de conocimientos.html

Guilar, M. (2009). Las ideas de Bruner: "de la revolución cognitiva" a la "revolución cultural". Educere, 13(44), 235-241. http://www.redalyc.org/pdf/356/35614571028.pdf

Hervás, R.; P. Miralles (2006). La importancia de enseñar a pensar en el aprendizaje de la historia. Educar en el 2000, (10), 34-40. http://www.educarm.es/documents/246424/461840/revsita9 art06.pdf/3716129f-b19d-4e2b-84d3-27bfaec09942

Lamoneda, M. (1998). ¿Cómo enseñamos historia en el nivel medio superior? Revista Mexicana de Investigación Educativa, 3(5), 101-112. http://www.comie.org.mx/v1/revista/visualizador.php?articulo=ART00239\&criterio=http://www.comie.org.mx/documentos/rmie/v03/ n005/pdf/rmiev03n05scC00n05es.pdf

Latapí, P. (2014). La investigación sobre la enseñanza de la historia en México. Querétaro: Universidad Autónoma de Querétaro.

Le Goff, J.; A. Santoni. (1996). Investigación y enseñanza de la historia. Michoacán: Instituto Michoacano de la Educación.

Lerner, V. (comp.) (1990). La enseñanza de Clío. Prácticas y propuestas para una didáctica de la historia. México: UNAM/Instituto Mora.

Linares, A. (Bienio 2007-2008). Desarrollo cognitivo: las teorías de Piaget y de Vygotsky. Master en Paidopsiquiatría. Barcelona: España: Universidad de Barcelona. http://www.paidopsiquiatria.cat/archivos/teorias desarrollo cognitivo 07-09 m1.pdf

Mckinry, W. L.; I. Westbury (1975). Stability and Change: The Public Schools of Gary, Indiana, 1940-1970. En Reid, W. A.; D. F. Walker (eds.). Case Studies in Curriculum Change: Great Britain and the United States. Londres: Routledge \& Kegan Paul, 1-53.

Pagés, J. (1994). La didáctica de las Ciencias Sociales, el currículum de Historia y la formación del profesorado. Signos. Teoría y Práctica de la Educación, (13), 38-51. https://historia1imagen.files.wordpress.com/2011/10/pages-la-didactica-de-las-cs-sociales-el-curriculum-dehistoria-y-la-formacion-de-profesorado.pdf

Pagés, J. (2000). La didáctica de las ciencias sociales en la formación inicial del profesorado. Iber, (24), 33-44. https://historia1imagen.files.wordpress.com/2011/12/pagc3a8s-j-2000-la-didc3a1ctica-de-las-ciencias-sociales-en-la-formacic3b3n-inicial-del-profesorado-iber-nc2ba24-abril-2000-33-44.pdf

Pagés, J. (2004). Enseñar a enseñar historia: la formación didáctica de los futuros profesores de historia". En Nicolás, E.; J. Goméz. Miradas a la historia: reflexiones historiográficas en recuerdo de Miguel Rodríguez Llopis. Murcia: Ediciones de la Universidad de Murcia, 155-178.

Pagés, J. (2012). La formación del profesorado de historia y ciencias sociales para la práctica reflexiva. Nuevas Dimensiones. Revista Electrónica de Didáctica de las Ciencias Sociales, (3), 
5-14. https://es.scribd.com/document/124135728/La-formacion-del-profesorado-de-historia-y-ciencias-sociales-para-la-practica-reflexiva-Pages-Joan-en-Revista-Electronica-deDidactica-de-las-Cienc

Patiño, L. (2007). Aportes del enfoque histórico cultural para la enseñanza. Educación y educadores, 10(1), 53-60. http://www.scielo.org.co/pdf/eded/v10n1/v10n1a05.pdf

Plá, S. (2005). Aprender a pensar históricamente. La escritura de la historia en Bachillerato. México: Plaza y Valdés / Colegio Madrid.

Plá, S. (2008). Metamorfosis del discurso histórico escolar: de la historia a la psicología en la reforma educativa de 1993 en México. Revista de Teoría y Didáctica de las Ciencias Sociales. (13), 171-194. https://www.redalyc.org/pdf/652/65216719009.pdf

Plá, S. (2012). La enseñanza de la historia como objeto de investigación. Secuencia (84), 163-184. https://www.academia.edu/1978237/La enseñanza de la historia como objeto de investigación Teaching History as an Object of Research

Plá, S. (2014). Ciudadanía y competitividad en la enseñanza de la historia. Los casos de México, Argentina y Uruguay. México: Universidad Iberoamericana.

Prats, J. (1989). Las experiencias didácticas como alternativas al cuestionario oficial: reflexiones críticas sobre las experiencias de "Germanía-75" "Historia 13-16". En Carretero, M.; J. I. Pozo; M. Asensio (comps.). La enseñanza de las Ciencias Sociales. Madrid: Visor, 201-210.

Prats, J.; R. Valls (2011). La didáctica de la historia en España: estado reciente de la cuestión. Didáctica de las Ciencias Experimentales y Sociales, (25), 17-35. http://www.usfx.bo/nueva/ vicerrectorado/citas/SOCIALES 8/Historia/Prats, J., \& Valls, R.pdf

Sallés, N. (2011). La enseñanza de la historia a través del aprendizaje por descubrimiento: evolución del proyecto treinta años después. Enseñanza de las Ciencias Sociales, (10), 3-10. http:// www.redalyc.org/pdf/3241/324127610002.pdf

Sánchez, A. (2006) Reflexiones sobre la historia que se enseña. En Galván, L. E. (coord.). La formación de una conciencia histórica. La enseñanza de la historia en México. México: Academia Mexicana de la Historia, 40-62.

Santisteban, A. (2017). Del tiempo histórico a la conciencia histórica: cambios en la enseñanza y el aprendizaje de la historia de los últimos 25 años. Diálogo Andino, (53), 87-99. https:// scielo.conicyt.cl/scielo.php?pid=S0719-2y812017000200087\&script=sci abstract\&tlng=es

Shemilt, D. (1987). El proyecto Historia 13-16 del Schools Council: pasado, presente y futuro. La geografía y la historia dentro de las ciencias sociales: hacia un currículum integrado. Madrid: MEC.

Waldegg, G.; D. Block (1996) Estudios de didáctica. México: COMIE / Grupo Editorial Iberoamérica. Zavala, A. (2015). Pensar "teóricamente" la práctica en la enseñanza de la historia. Historia Hoje, 4(8), 181-203. https://www.researchgate.net/publication/295085454 Pensar 'teoricamente' la practica de la ensenanza de la historia 